\author{
Marek Gawel \\ Jürgen Aschoff \\ Arne May \\ Bruce R. Charlesworth \\ on behalf of the REALIZE \\ Study Group
}

\section{Treatment satisfaction with zolmitriptan nasal spray for migraine in a real life setting: results from phase two of the REALIZE study}

Received: 24 March 2005

Accepted in revised form: 7 July 2005

Published online: 21 September 2005

M. Gawel (两)

Department of Medicine (Neurology), Sunnybrook and Women's Health

Sciences Centre,

Suite 515, 60 Grosvenor Street,

Toronto, Ontario M5S 1B6, Canada

e-mail: marek.gawel@sw.ca

J. Aschoff

Department of Neurology,

University of Ulm, Ulm, Germany

A. May

Department of Neurology,

University of Regensburg,

Regensburg, Germany

B.R. Charlesworth

AstraZeneca,

Macclesfield, UK

\begin{abstract}
In phase one of the
REALIZE study, zolmitriptan nasal spray demonstrated a significant headache response from $10 \mathrm{~min}$ post-dose and total symptom relief from $30 \mathrm{~min}$ post-dose. The objective of phase two was to investigate patients' dosing patterns, satisfaction and preference following openlabel treatment with the nasal spray. Up to 3 attacks were treated. The ITT population consisted of 851 patients. The median time from onset of symptoms to treatment was $1 \mathrm{~h} 15 \mathrm{~min}$ (primary endpoint). Most patients reported being satisfied or very satisfied with zolmitriptan nasal spray (75.7\%). Furthermore, the majority of patients would be willing to use zolmitriptan nasal spray in the
\end{abstract}

future $(59.8 \%)$ and preferred zolmitriptan nasal spray over previous therapies (57.8\%). Zolmitriptan nasal spray was well tolerated. Most patients were satisfied with zolmitriptan nasal spray, were willing to continue using it and preferred it to previous therapies.

Key words Migraine $\cdot$ Zolmitriptan • Intranasal $\cdot$ Satisfaction

\section{Introduction}

Migraine is a common, recurrent neurological disorder characterised by headache that is often associated with gastrointestinal and autonomic symptoms [1,2]. The chronic and debilitating nature of migraine can impede patients' ability to carry out normal daily activities, thereby reducing quality of life $[3,4]$.

The 5-hydroxytryptamine $\left(5-\mathrm{HT}_{1 \mathrm{~B} / 1 \mathrm{D}}\right)$ agonists, collectively known as triptans, are a major advance in the treatment of migraine. The beneficial effects of the triptans in patients with migraine are related to multiple mechanisms of action at sites implicated in the pathophysiology of migraine.

Zolmitriptan is a $5-\mathrm{HT}_{1 \mathrm{~B} / 1 \mathrm{D}}$ receptor agonist that is highly effective for the acute treatment of migraine $[5,6]$. A 5-mg nasal spray formulation of zolmitriptan has recently been developed to offer patients a convenient alternative to oral tablets [7]. Nasal spray administration provides rapid absorption directly across the nasal mucosa, resulting in a fast onset of action [8]. Furthermore, nasal admin- 
istration may be preferable to conventional oral preparations for those patients who experience nausea and vomiting during migraine attacks, or those who have difficulty swallowing tablets.

The efficacy and tolerability of zolmitriptan nasal spray has been established in previous clinical trials [8,9]. In the Real Life Intranasal Zolmitriptan Experience (REALIZE) study, patients treated migraine attacks with zolmitriptan nasal spray without a strictly recommended dosing regimen [10]. This setting was designed to be more representative of clinical practice than most clinical trials, where patients are typically instructed to treat migraine attacks within a specified time period or when pain severity has reached a designated level. The REALIZE study consisted of two phases. In phase one, the efficacy of intranasal zolmitriptan compared with placebo in the treatment of a single migraine attack was investigated; the primary endpoint of the study was total symptom relief (defined as no headache pain, nausea, photophobia or phonophobia) at $1 \mathrm{~h}$ post-dose. This was significantly superior for zolmitriptan nasal spray from $30 \mathrm{~min}$ onwards. Zolmitriptan nasal spray also demonstrated a significant headache response from $10 \mathrm{~min}$ post-dose, confirming the very fast onset of this formulation [10]. In phase two, in which up to 3 migraine attacks were treated with open-label zolmitriptan nasal spray, patients' dosing patterns, satisfaction and preference were assessed. The results of the second phase of this study are reported here.

\section{Methods}

\section{Patients}

Patients aged 18-66 years with an established diagnosis of migraine (as defined by the International Headache Society Criteria [11]), with an age of onset of $<50$ years were eligible for inclusion in the study. Patients were required to have experienced at least one migraine attack per month in the previous 3 months and have the ability to differentiate between migraine and non-migraine headaches.

Exclusion criteria included: a history of basilar, ophthalmoplegic or hemiplegic migraine headache, or any potential serious neurological condition associated with headache; diagnosis or suspicion of drug-induced or chronic daily headaches; symptoms of, or significant risk factors for, cardiovascular disease; systolic blood pressure of $>150 \mathrm{mmHg}$ or diastolic blood pressure $>95$ $\mathrm{mmHg}$; any recent history of alcohol or drug abuse; severe hepatic impairment; or an unacceptable adverse experience following previous use of any 5-HT agonist. Use of monoamine oxidase A inhibitors, methysergide or methylergonovine was not allowed within the 2 weeks prior to randomisation.

The study was conducted in accordance with the Declaration of Helsinki and all investigators complied with Good Clinical
Practices. Written consent was obtained from each patient prior to participation in the study.

Study design

This multicentre, randomised, parallel-group, two-phase study was conducted in Canada and Germany between May 2002 and August 2003. In phase one of the study [10], patients were randomised to receive zolmitriptan $5 \mathrm{mg}$ nasal spray or placebo nasal spray in a double-blind fashion, to treat a single migraine attack (Visit 1). Patients were instructed to use diary cards to record efficacy data relating to the attack treated with study medication and were asked to return to the clinic within 7 days of the treated attack (Visit 2). Patients could then continue into the open-label second phase of the study (reported here), in which the next three migraine attacks were treated with zolmitriptan nasal spray. Patients could choose to take study medication at any time after symptom onset to treat a migraine attack with any severity of headache pain. Patients were permitted to use a second dose of zolmitriptan nasal spray or an agreed escape medication if pain relief had not been obtained by $2 \mathrm{~h}$ after the initial dose. Patients were required to return to the clinic within 14 days of treating the fourth migraine attack or 6.5 months after randomisation (Visit 3). At Visits 2 and 3, the investigator reviewed each migraine attack with the patient and ensured that diary card information had been clearly completed.

\section{Assessments}

\section{Efficacy}

The primary endpoint of the second phase of the study was the time between onset of headache pain and dosing with zolmitriptan nasal spray, as recorded in diary cards. Secondary endpoints included: the time to a second dose of zolmitriptan nasal spray or escape medication; patient satisfaction with zolmitriptan nasal spray; willingness to use the formulation to treat future migraine attacks; patient preference for zolmitriptan nasal spray over previous migraine therapies.

Satisfaction variables were assessed at the end of the study by asking the patient direct questions regarding overall satisfaction, and satisfaction with the speed of headache relief, ease of use, convenience and reliability of zolmitriptan nasal spray. Satisfaction was assessed using a 4-point Likert scale, where 1 indicated "dissatisfied", 2 indicated "not satisfied or dissatisfied", 3 indicated "satisfied" and 4 indicated "very satisfied". Patients were also asked to respond "strongly agree", "agree", "disagree" or "strongly disagree" to the following statements: (a) "I am willing to use zolmitriptan nasal spray for the treatment of future migraine attacks", and (b) "I prefer zolmitriptan nasal spray over the migraine therapies I used before the study".

\section{Safety}

Patients were encouraged to report any adverse events experienced at any time during phase two of the study in the diary cards. Any detrimental changes in the patient's condition after 
study entry were only recorded as adverse events if occurring during the first $24 \mathrm{~h}$ after taking the study drug (a period of at least 5 times the half-life of zolmitriptan), although serious adverse events were recorded throughout the study. The safety of zolmitriptan nasal spray after repeated use in this study was assessed at the end of the study from the diary card data.

\section{Statistical methods}

The safety population included all patients who treated a migraine attack with zolmitriptan nasal spray in phase two of the study. The intention-to-treat (ITT) population comprised those patients in the safety population who provided post-treatment efficacy data.

Data on dosing patterns, patient satisfaction with zolmitriptan nasal spray, willingness to continue using zolmitriptan nasal spray and preference for zolmitriptan nasal spray over previous migraine therapies were summarised using descriptive statistical methods only. These data were not subjected to any formal statistical analysis. All adverse event data were summarised using Medical Dictionary for Regulatory Activities (MEDRA) preferred terms and system organ classes.

\section{Results}

\section{Patients}

Of the 915 patients who treated a migraine attack with zolmitriptan nasal spray or placebo in phase one, 886 patients $(96.8 \%)$ entered phase two of the study. A total of 853 patients treated at least one migraine attack with zolmitriptan nasal spray during phase two of the study (safety population), with 851 of these patients providing posttreatment efficacy data (ITT population). Demographic characteristics of the ITT population are shown in Table 1.

Table 1 Demographic characteristics of the intention-to-treat population $(n=851)$

\begin{tabular}{lrr}
\hline Characteristic & \multicolumn{2}{c}{ Value } \\
\hline Gender, $n(\%)$ & 106 & $(12.5)$ \\
$\quad$ Male & 745 & $(87.5)$ \\
$\quad$ Female & & \\
Race, $n(\%)$ & 5 & $(0.6)$ \\
$\quad$ Black & 839 & $(98.6)$ \\
$\quad$ Caucasian & 4 & $(0.5)$ \\
$\quad$ Oriental & 3 & $(0.4)$ \\
$\quad$ Other & 41.9 & $(18-66)$ \\
Age, years (range) & 167.5 & $(148-198)$ \\
Height, cm (range) & 69.8 & $(40-136)$ \\
Weight, kg (range) & & \\
\hline
\end{tabular}

As expected from phase one, the phase two study population was representative of the general migraine population (predominantly female with a mean age of about 40 years). A total of 760 patients ( $89.3 \%$ of the ITT population) used zolmitriptan nasal spray to treat 3 migraine attacks; 55 patients $(6.5 \%)$ treated 2 attacks and 36 patients $(4.2 \%)$ treated one attack. In total, 2426 migraine attacks were treated with zolmitriptan nasal spray.

\section{Efficacy}

The median time from the onset of symptoms to treatment with zolmitriptan nasal spray was 1 h 15 min (mean 2 h 42 min; range: $0-53 \mathrm{~h}$ ). Five hundred and eighteen attacks $(21.5 \%)$ were treated in the first $15 \mathrm{~min}, 328$ (13.6\%) from $>15$ to $30 \mathrm{~min}, 97(4.0 \%)$ from $>30$ to $45 \mathrm{~min}, 181(7.5 \%)$ from $>45$ to $60 \mathrm{~min}$, and $1286(53.4 \%)$ after $60 \mathrm{~min}$. Pain intensity at the onset of the attack and immediately before taking study medication is shown in Table 2. Although $33 \%$ of attacks were associated with mild pain at onset, only $7 \%$ of attacks were treated while pain was mild. Additionally, although $39 \%$ of attacks were associated with severe pain when treated, only $19 \%$ were of severe pain intensity at the onset of the attack. Almost half the attacks were of moderate pain intensity at onset, and just over half were treated with zolmitriptan nasal spray when pain intensity was moderate. As the intensity of headache pain at onset increased, the median time taken to treat the attack decreased (Fig. 1a). However, median times to treatment were longer in attacks with initially mild or moderate pain that had worsened by the time of treatment (Fig. 1b), suggesting that pain worsened when treatment was delayed.

Of the 2426 migraine attacks treated with zolmitriptan nasal spray, $1108(45.7 \%)$ were followed by either a second dose of zolmitriptan nasal spray or an agreed escape medication. The mean time to use of further medication was 6 h 34 min (range: 0-24 h).

In response to the overall satisfaction question ("How satisfied are you overall with the nasal spray medication you have used to treat your migraines in this study?"), 644 $(75.7 \%)$ patients reported being "satisfied" or "very satisfied" with zolmitriptan nasal spray; 150 (17.6\%) were "not satisfied or dissatisfied", $46(5.4 \%)$ were "dissatisfied" and data were missing for $11(1.3 \%)$ patients (Fig. 2). Over $75 \%$ of patients reported being "satisfied" or "very satisfied" with zolmitriptan nasal spray for each of the following: speed of relief $(646 ; 75.9 \%)$; ease of use $(779 ; 91.5 \%)$; convenience $(750 ; 88.1 \%)$; reliability $(644$; $75.7 \%$ ) (Fig. 3).

In response to the question "I am willing to use zolmitriptan nasal spray for the treatment of future 
Table 2 Intensity of migraine headache at the onset of the attack and immediately prior to taking study medication in an open-label study in which 851 patients could treat up to 3 attacks (attacks 2, 3 and 4) with zolmitriptan nasal spray. Patients could choose when to take zolmitriptan nasal spray in terms of severity of the pain

Time window and headache

intensity

Migraine attacks treated with zolmitriptan nasal spray ${ }^{\mathrm{a}}$

\begin{tabular}{rrrr}
\hline Attack 2 & Attack 3 & Attack 4 & Total \\
$(n=851), n(\%)$ & $(n=815), n(\%)$ & $(n=760), n(\%)$ & $(n=2426), n(\%)$ \\
\hline
\end{tabular}

\begin{tabular}{lrrrr}
\hline Onset of attack & & & & \\
$\quad$ Mild & $296(34.8)$ & $250(30.7)$ & $250(32.9)$ & $796(32.8)$ \\
$\quad$ Moderate & $415(48.8)$ & $403(49.4)$ & $345(45.4)$ & $1163(47.9)$ \\
$\quad$ Severe & $138(16.2)$ & $160(19.6)$ & $164(21.6)$ & $462(19.0)$ \\
$\quad$ Not recorded & $2(0.2)$ & $2(0.2)$ & & $(0.1)$ \\
Immediately prior to taking & & & & \\
zolmitriptan nasal spray & & & & \\
$\quad$ Mild & $58(6.8)$ & $58(7.1)$ & $46(6.1)$ & $162(6.7)$ \\
$\quad$ Moderate & $496(58.3)$ & $434(53.3)$ & $390(51.3)$ & $1320(54.4)$ \\
$\quad$ Severe & $296(34.8)$ & $320(39.3)$ & $319(42.0)$ & $935(38.5)$ \\
$\quad$ Not recorded & $1(0.1)$ & $3(0.4)$ & $5(0.7)$ & $9(0.4)$ \\
\hline
\end{tabular}

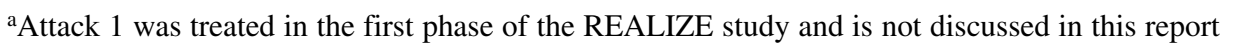

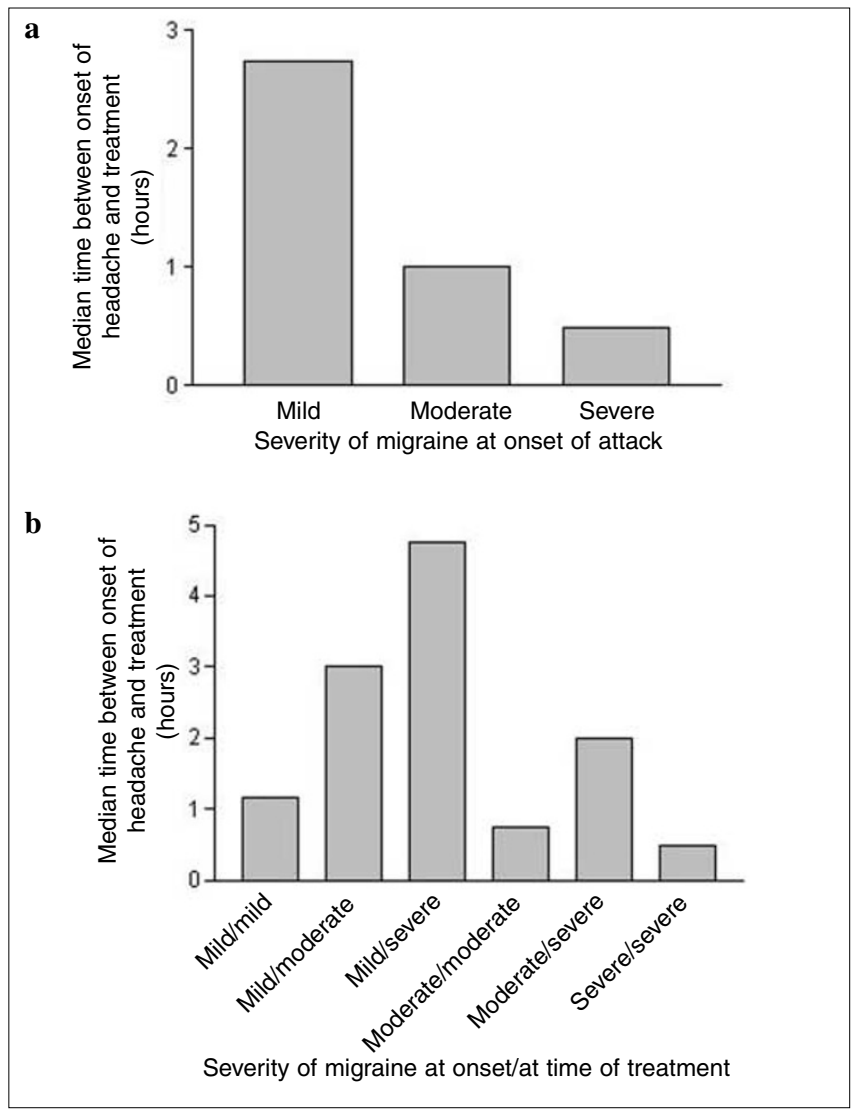

Fig. 1 Median time taken between onset of headache and treatment with zolmitriptan nasal spray for (a) 2393 migraine attacks that were mild, moderate or severe at onset and treated within $24 \mathrm{~h}$ of onset and (b) 2372 migraine attacks that were mild, moderate or severe at onset and had either intensified or remained at the same intensity at the time of treatment

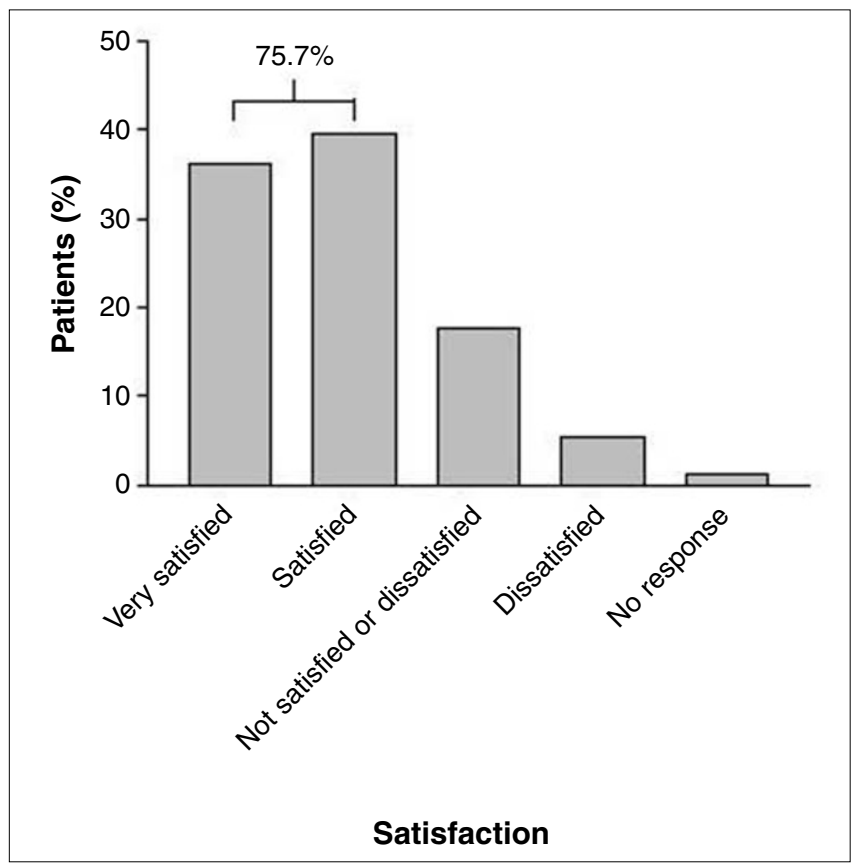

Fig. 2 Overall patient satisfaction with zolmitriptan nasal spray following treatment of up to 3 migraine attacks in an open-label study. Patients were asked "How satisfied are you overall with the nasal spray you have used to treat your migraines in this study?" $(n=851)$

migraine attacks", 509 patients (59.8\%) "agreed" or "strongly agreed", 216 (25.4\%) "disagreed" and 115 $(13.5 \%)$ "strongly disagreed". Similarly, in response to the question "I prefer zolmitriptan nasal spray over the migraine therapies I used before the study", 492 (57.8\%) "agreed" or "strongly agreed", 245 (28.8\%) "disagreed" and $103(12.1 \%)$ "strongly disagreed". 


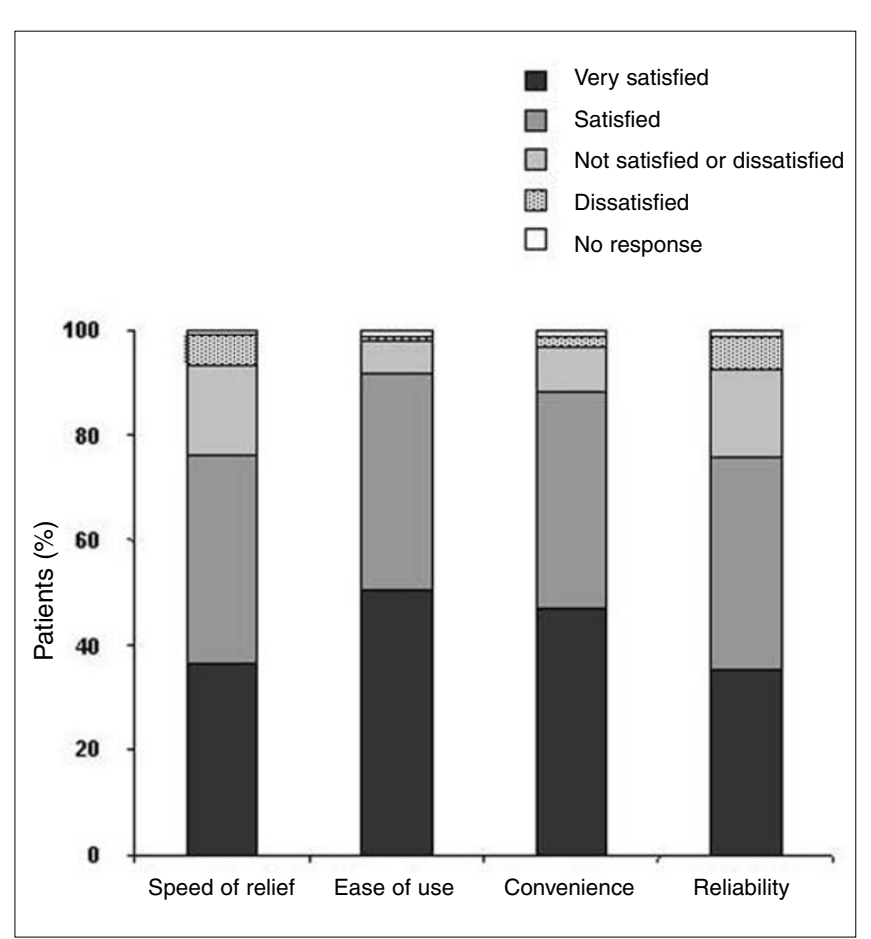

Fig. 3 Patient satisfaction with the speed of relief, ease of use, convenience and reliability of zolmitriptan nasal spray following treatment of up to 3 migraine attacks in an open-label study $(n=851)$

Safety

Overall, adverse events occurred in 430 (50.4\%) zolmitriptan nasal spray recipients and in 967 (39.9\%) attacks treated with zolmitriptan nasal spray. The majority of these events were of mild or moderate intensity and resolved without intervention. Of the 644 patients who reported being "satisfied" or "very satisfied" with zolmitriptan nasal spray, almost half (48\%) experienced adverse events. Serious adverse events were reported in 2 patients (subileus occurring during treatment of a migraine attack in one patient, and Norwalk virus gastroenteritis occurring after treatment of an attack in one patient). Only 12 patients $(1.4 \%)$ experienced adverse events that led to premature discontinuation from the study; these were: cardiovascular disorder (not otherwise specified), tachycardia (not otherwise specified), nausea, fatigue, malaise, hunger, burning sensation (not otherwise specified), dizziness, dysgeusia, hyperaesthesia, hypoaesthesia, paraesthesia, somnolence, tremor, anxiety, oropharyngeal swelling, throat irritation and hypertension (not otherwise specified). Adverse events occurring in $\geq 2 \%$ of attacks included dysgeusia (which was reported in $15.1 \%$ of attacks, but led to treatment discontinuation in only 2 patients $(0.2 \%)$ ), throat irritation $(5.8 \%$ of attacks), nasal passage irritation (4.6\%), dizziness (4.6\% of attacks) and fatigue $(3.4 \%)$.

\section{Discussion}

The main focus of the second phase of the REALIZE study was patient experience with zolmitriptan nasal spray without a dictated regimen for dosing. Patients were able to treat migraine attacks of any intensity, without a fixed time between symptom onset and dosing. Hence, this provided an important indication of patients' dosing patterns with zolmitriptan nasal spray in a true clinical practice setting. The open-label treatment of three attacks was deemed necessary to allow patients to become accustomed to nasal administration, before making any valid judgements about satisfaction or preference.

The results of the study indicate that most patients treat at either $<30 \mathrm{~min}(35 \%)$ or $>60 \mathrm{~min}(53 \%)$ after attack onset. Only a small proportion of attacks were treated while pain was mild (7\%), even though $33 \%$ of attacks were of mild pain intensity at onset, indicating that a substantial proportion of patients were reluctant to treat when pain intensity was mild. A relatively high proportion of migraine attacks were treated when pain was severe (39\%), although only $19 \%$ of attacks began with severe pain, indicating that when patients waited to treat, headache pain often intensified. The results of this study suggest that the greater the intensity of pain at onset of the attacks, the sooner the attack is treated. Clinical studies have shown that treating a migraine early, while pain is mild, results in the best response to therapy [12-14]. Data reported by Klapper et al. demonstrated the efficacy of zolmitriptan conventional tablets, taken while headache pain was mild, in terms of headache response and reduction in progression to more severe pain intensity [15].

Patient satisfaction with regard to ease of use and convenience of zolmitriptan nasal spray was high in the second phase of the REALIZE study, with $88 \%-92 \%$ of patients "satisfied" or "very satisfied" with these aspects of treatment. If patients are made aware of the potential benefits of treating before pain intensifies, the ease of use and convenience that patients associate with zolmitriptan nasal spray could help to ensure that patients use the treatment in a way that achieves optimal efficacy.

Regardless of when patients chose to treat a migraine attack, approximately three quarters (76\%) were "very satisfied" or "satisfied" with zolmitriptan nasal spray overall. These results follow on from phase one of the REALIZE study, which demonstrated the efficacy and fast onset of action of zolmitriptan nasal spray [10]. In the present study, the majority of patients (about 76\%) were "very satisfied" or "satisfied" with zolmitriptan nasal spray with regard to speed of relief and reliability. Such attributes have been previously identified as being important characteristics of an acute migraine therapy [16-18]. Furthermore, the majority of patients would be willing to 
use zolmitriptan nasal spray in the future and preferred zolmitriptan nasal spray over previous therapies.

Treatment satisfaction with zolmitriptan nasal spray has also been reported in two studies conducted at different clinics in Sweden [19, 20]. The first study was based on patients' cumulative experience of using the nasal spray to treat up to 6 consecutive migraine attacks [19]. The majority of the 232 patients $(69 \%)$ wished to continue treatment with the nasal spray at the end of the study. The most common reasons given for wanting to continue using zolmitriptan nasal spray were: fast onset of action (75\%); only needing one dose (58\%); and achieving complete relief from migraine pain $(56 \%)$. The second study involved 117 patients who had tried zolmitriptan nasal spray on at least one occasion for the treatment of migraine [20]. Seventy percent of patients wished to continue using zolmitriptan nasal spray; the main reasons were effectiveness and fast onset of action (complete relief from headache pain was achieved within one hour in $65 \%$ of patients). Patient satisfaction was also assessed as a secondary endpoint in a long-term safety and efficacy trial of zolmitriptan nasal spray; overall satisfaction was rated as "excellent" or "good" by most patients [9].
Zolmitriptan nasal spray was well tolerated in phase two of the REALIZE study, with very few patients withdrawing from the study due to adverse events. The most common adverse event, dysgeusia, was typically reported as mild and did not appear to be troublesome to patients.

\section{Conclusions}

The satisfaction data from the present study demonstrate that zolmitriptan nasal spray is effective in the acute treatment of migraine and preferred to other therapies by the majority of patients. The performance of zolmitriptan nasal spray in the clinical practice setting suggests that it offers patients and physicians a significant advance in migraine therapy.

Acknowledgements The authors would like to thank Neil Marmont for editorial support in the preparation of the manuscript. AstraZeneca provided financial support for this editorial assistance.

\section{References}

1. Lipton RB, Stewart WF, Diamond S, Diamond ML, Reed M (2001) Prevalence and burden of migraine in the United States: data from the American Migraine Study II. Headache 41:646-657

2. Dahlöf CG, Solomon GD (1998) The burden of migraine to the individual sufferer: a review. Eur J Neurol 5(6):525-533

3. Terwindt GM, Ferrari MD, Tijhuis M, Groenen SM, Picavet HS, Launer LJ (2000) The impact of migraine on quality of life in the general population. The GEM study. Neurology 55:624-629

4. Solomon GD, Santanello N (2000) Impact of migraine and migraine therapy on productivity and quality of life. Neurology 55[Suppl 2]:S29-35

5. Dahlöf C, Diener HC, Goadsby PJ, Massiou H, Olesen J, Schoenen J, Wilkinson M, Sweet RM, Klein KB (1998) Zolmitriptan, a 5-HT $1 \mathrm{~B} / 1 \mathrm{D}$ receptor agonist for the acute oral treatment of migraine: a multicentre, dose-range finding study. Eur J Neurol 5:535-543
6. Rapoport AM, Ramadan NM, Adelman JU, Mathew NT, Elkind AH, Kudrow DB, Earl NL (1997) Optimizing the dose of zolmitriptan for the acute treatment of migraine. Neurology 49:1210-1218

7. Dowson AJ, Charlesworth B (2002) Review of zolmitriptan and its clinical applications in migraine. Expert Opin Pharmacother 3(7):993-1005

8. Charlesworth BR, Dowson AJ, Purdy A, Becker WJ, Boes-Hansen S, Farkkila M (2003) Speed of onset and efficacy of zolmitriptan nasal spray in the acute treatment of migraine. CNS Drugs 17(9):653-667

9. Dowson AJ, Charlesworth BR, Purdy A, Becker WJ, Boes-Hansen S, Farkkila M (2003) Tolerability and consistency of effect of zolmitriptan nasal spray in a long-term migraine treatment trial. CNS Drugs 17:839-851

10. Gawel M, Aschoff A, May A, Charlesworth BR (2005) Zolmitriptan $5 \mathrm{mg}$ nasal spray has a very fast onset of action and sustained efficacy: results from the REALIZE study. Headache 45(1):7-16
11. Headache Classification Committee of the International Headache Society (1988) Classification and diagnostic criteria for headache disorders, cranial neuralgias and facial pain. Cephalalgia 8[Suppl 7]:1-96

12. International 311C90 Long-Term Study Group (1998) The long-term tolerability and efficacy of oral zolmitriptan (Zomig, 311C90) in the acute treatment of migraine. Headache 38:173-183

13. Cady RK, Sheftell F, Lipton RB, O'Quinn S, Pharmd, Jones M, Putnam DG, Crisp A, Metz A, McNeal S (2000) Effect of early intervention with sumatriptan on migraine pain: retrospective analyses of data from three clinical trials. Clin Ther 22:1035-1037

14. Winner P, McNeal S, O'Quinn S, Metz, A (2002) Treatment of migraine at the first sign of pain: prospective, doubleblind, placebo-controlled, multicenter studies of sumatriptan $50 \mathrm{mg}$ and 100 $\mathrm{mg}$ versus placebo. Neurology 58[Suppl 3]:A415 
15. Klapper J, Lucas C, Røsjø Ø, Charlesworth B, ZODIAC study group (2004) Benefits of treating highly disabled migraine patients with zolmitrip$\tan$ while pain is mild. Cephalalgia 24(11):918-924

16. Lipton RB, Hamelsky SW, Dayno JM (2002) What do patients with migraine want from acute migraine treatment? Headache 42[Suppl 1]:3-9
17. Pascual J, Munoz R, Leira R (2001) An open preference study with sumatriptan $50 \mathrm{mg}$ and zolmitriptan $2.5 \mathrm{mg}$ in 100 migraine patients. Cephalalgia 21:680-684

18. Ryan RE (2001) Patient treatment preferences and the 5- $\mathrm{HT}_{1 \mathrm{~B} / 1 \mathrm{D}}$ agonists. Arch Intern Med 161:2545-2553
19. Dahlöf CG, Linde M, Kerekes E (2004) Zolmitriptan nasal spray provides fast relief of migraine symptoms and is preferred by patients: a Swedish study of preference in clinical practice. J Headache Pain 5:237-242

20. Dahlöf CG, Boes-Hansen S, Dowson A (2003) Zolmitriptan nasal spray improves patient satisfaction by providing fast and effective relief from migraine symptoms. Cephalalgia 23:709 (Abstract P5N67) 